\title{
The electron energy loss rate due to radiative recombination ${ }^{\star}$
}

\author{
Junjie Mao ${ }^{1,2}$, Jelle Kaastra ${ }^{1,2}$, and N. R. Badnell ${ }^{3}$ \\ ${ }^{1}$ SRON Netherlands Institute for Space Research, Sorbonnelaan 2, 3584 CA Utrecht, The Netherlands \\ e-mail: J.Mao@sron.nl \\ 2 Leiden Observatory, Leiden University, Niels Bohrweg 2, 2300 RA Leiden, The Netherlands \\ 3 Department of Physics, University of Strathclyde, Glasgow G4 0NG, UK \\ Received 13 September 2016 / Accepted 2 December 2016
}

\begin{abstract}
Context. For photoionized plasmas, electron energy loss rates due to radiative recombination (RR) are required for thermal equilibrium calculations, which assume a local balance between the energy gain and loss. While many calculations of total and/or partial RR rates are available from the literature, specific calculations of associated RR electron energy loss rates are lacking.

Aims. Here we focus on electron energy loss rates due to radiative recombination of H-like to Ne-like ions for all the elements up to and including zinc $(Z=30)$, over a wide temperature range.

Methods. We used the AUTOSTRUCTURE code to calculate the level-resolved photoionization cross section and modify the ADASRR code so that we can simultaneously obtain level-resolved RR rate coefficients and associated RR electron energy loss rate coefficients. We compared the total RR rates and electron energy loss rates of H I and He I with those found in the literature. Furthermore, we utilized and parameterized the weighted electron energy loss factors (dimensionless) to characterize total electron energy loss rates due to RR.

Results. The RR electron energy loss data are archived according to the Atomic Data and Analysis Structure (ADAS) data class adf48. The RR electron energy loss data are also incorporated into the SPEX code for detailed modeling of photoionized plamsas.
\end{abstract}

Key words. atomic data - atomic processes

\section{Introduction}

Astrophysical plasmas observed in the X-ray band can roughly be divided into two subclasses: collisional ionized plasmas and photoionized plasmas. Typical collisional ionized plasmas include stellar coronae (in coronal/collisional ionization equilibrium), supernova remnants (SNRs, in nonequilibrium ionization) and the intracluster medium (ICM). In low-density, high-temperature collisional ionized plasma, for example, ICM, collisional processes play an important role (for a review see e.g., Kaastra et al. 2008). In contrast, in a photoionized plasma, photoionization, recombination and fluorescence processes are important in addition to collisional processes. Both the equations for the ionization balance (also required for a collisional ionized plasma) and the equations of the thermal equilibrium are used to determine the temperature of the photoionized plasma. Typical photoionized plasmas in the X-ray band can be found in X-ray binaries (XRBs) and active galactic nuclei (AGN).

For collisional ionized plasmas, various calculations of total radiative cooling rates are available in the literature, such as Cox \& Daltabuit (1971), Raymond et al. (1976), Sutherland \& Dopita (1993), Schure et al. (2009), Foster et al. (2012), and Lykins et al. (2013). These calculations take advantage of full plasma codes, such as SPEX (Kaastra et al. 1996) and APEC (Smith et al. 2001), and do not treat individual energy loss (cooling) processes separately. Total radiative cooling rates include the energy loss of both the line emission and continuum emission. The latter includes the energy loss due to radiative recombination (RR). Even more specifically, the energy loss due

\footnotetext{
* Full Tables 1 and 2 are available at the CDS via anonymous ftp to cdsarc.u-strasbg. fr (130.79.128.5) or via

http://cdsarc.u-strasbg.fr/viz-bin/qcat?]/A+A/599/A10
}

to RR can be separated into the electron energy loss and ion energy loss.

On the other hand, for photoionized plasmas, the electron energy loss rate due to RR is one of the fundamental parameters for thermal equilibrium calculations, which assume a local balance between the energy gain and loss. Energy can be gained via photoionization, Auger effect, Compton scattering, collisional ionization, collisional de-excitation and so forth. Energy loss can be due to, for example, radiative recombination, dielectronic recombination, three-body recombination, inverse Compton scattering, collisional excitation, and bremsstrahlung, as well as the line/continuum emission following these atomic processes. In fact, the energy loss and gain of all these individual processes need to be known. The calculations of electron energy loss rates due to RR in the Cloudy code (Ferland et al. 1998, 2013) are based on hydrogenic results (Ferland et al. 1992; LaMothe \& Ferland 2001). In this manuscript, we focus on improved calculations of the electron energy loss due to radiative recombination, especially providing results for He-like to $\mathrm{Ne}$ like isoelectronic sequences.

While several calculations of RR rates, including total rates and/or detailed rate coefficients, for different isoelectronic sequences are available, for example, Gu (2003) and Badnell (2006), specific calculations of the associated electron energy loss rate due to RR are limited. The pioneering work was carried out by Seaton (1959) for hydrogenic ions using the asymptotic expansion of the Gaunt factor for photoionization cross sections (PICSs).

By using a modified semiclassical Kramers formula for radiative recombination cross sections (RRCSs), Kim \& Pratt (1983) calculated the total RR electron energy loss rate for a few ions in a relatively narrow temperature range. 
Ferland et al. (1992) used the $n l$-resolved hydrogenic PICSs provided by Storey \& Hummer (1991) to calculate both $n$-resolved RR rates $\left(\alpha_{i}^{\mathrm{RR}}\right)$ and electron energy loss rates $\left(L_{\mathrm{i}}^{\mathrm{RR}}\right)$. Contributions up to and including $n=1000$ are taken into account.

Using the same $n l$-resolved hydrogenic PICSs provided by Storey \& Hummer (1991), Hummer (1994) calculated the RR electron energy loss rates for hydrogenic ions in a wide temperature range. In addition, Hummer \& Storey (1998) calculated PICSs of He I (photoionizing ion) for $n \leq 25$ with their close-coupling $\boldsymbol{R}$-matrix calculations. Together with hydrogenic (Storey \& Hummer 1991) PICSs for $n>25$ (up to $n=800$ for low temperatures), the RR electronic energy loss rate coefficient of He I (recombined ion) was obtained.

Later, LaMothe \& Ferland (2001) used the exact PICSs from the Opacity Project (Seaton et al. 1992) for $n<30$ and PICSs of Verner \& Ferland (1996) for $n \geq 30$ to obtain $n$-resolved RR electron energy loss rates for hydrogenic ions in a wide temperature range. The authors introduced the ratio of $\beta / \alpha$ (dimensionless), where $\beta=L / k T$ and $L$ is the RR electron energy loss rate. The authors also pointed out that $\beta / \alpha$ changes merely by 1 dex in a wide temperature range; meanwhile $\alpha$ and $\beta$ change more than 12 dex.

In the past two decades, more detailed and accurate calculations of PICSs of many isoelectronic sequences have been carried out (e.g., Badnell 2006), which can be used specifically to calculate the electron energy loss rates due to RR.

Currently, in the SPEX code (Kaastra et al. 1996), the assumption that the mean kinetic energy of a recombining electron is $3 k T / 4$ (Kallman \& McCray 1982) is applied for calculating the electron energy loss rate due to RR. Based on the level-resolved PICSs provided by the AUTOSTRUCTURE ${ }^{1}$ code (v24.24.3; Badnell 1986), the electron energy loss rates due to RR are calculated in a wide temperature range for the $\mathrm{H}$-like to Ne-like isoelectronic sequences for elements up to and including $\mathrm{Zn}(Z=30)$. Subsequently, the electron energy loss rate coefficients $(\beta=L / k T)$ are weighted with respect to the total RR rates $\left(\alpha_{\mathrm{t}}\right)$, yielding the weighted electron energy loss factors ( $f=\beta / \alpha_{\mathrm{t}}$, dimensionless). The weighted electron energy loss factors can be used, together with the total RR rates, to update the description of the electron energy loss due to RR in the SPEX code or other codes.

In Sect. 2, we describe the details of the numerical calculation from PICSs to the electron energy loss rate due to RR. Typical results are shown graphically in Sect. 3. Parameterization of the weighted electron energy loss factors is also illustrated in Sect. 3. The detailed RR electron energy loss data are archived according to the Atomic Data and Analysis Structure (ADAS) data class adf48. Full tabulated (unparameterized and parameterized) weighted electron energy loss factors are available in CDS. Comparison of the results for $\mathrm{HI}$ and He I can be found in Sect. 4.1. The scaling of the weighted electron energy loss factors with respect to the square of the ionic charge of the recombined ion can be found in Sect. 4.2. We also discuss the electron and ion energy loss due to RR (Sect. 4.3) and the total RR rates (Sect. 4.4).

Throughout this paper, we refer to the recombined ion when we speak of the radiative recombination of a certain ion, since the line emission following the radiative recombination comes from the recombined ion. Furthermore, only RR from the ground level of the recombining ion is discussed here.

\footnotetext{
1 http://amdpp.phys.strath.ac.uk/autos/
}

\section{Methods}

\subsection{Cross sections}

The AUTOSTRUCTURE code is used for calculating levelresolved nonresonant PICSs under the intermediate coupling (IC) scheme (Badnell \& Seaton 2003). The atomic and numerical details can be found in Badnell (2006); we briefly state the main points here. We use the Slater-type-orbital model potential to determine the radial functions. We calculated PICSs first at zero kinetic energy of the escaping electron. Subsequently, we calculated them on a $z$-scaled logarithmic energy grid with three points per decade, ranging from $\sim z^{2} 10^{-6}$ to $z^{2} 10^{2}$ ryd, where $z$ is the ionic charge of the photoionizing ion/atom. PICSs at even higher energies are at least several orders of magnitude smaller compared to PICSs at zero kinetic energy of the escaping electron. Nonetheless, it still can be important, especially for the $s$ - and $p$-orbit, to derive the RR data at the high temperature end. We take advantage of the analytical hydrogenic PICSs (calculated via the dipole radial integral; Burgess 1965) and scale them to the PICS with the highest energy calculated by AUTOSTRUCTURE to obtain PICSs at very high energies. Fully $n L S J$-resolved PICSs for those levels with $n \leq 15$ and $l \leq 3$ are calculated specifically. For the rest of the levels, we use the fast, accurate and recurrence hydrogenic approximation (Burgess 1965). Meanwhile, bundled- $n$ PICSs for $n=16,20,25,35,45,55,70,100,140,200,300,450,700$, and 999 are also calculated specifically to derive the total RR and electron energy loss rates (interpolation and quadrature required as well).

The inverse process of dielectronic and radiative recombination is resonant and nonresonant photoionization, respectively. Therefore, radiative recombination cross sections (RRCSs) are obtained through the Milne relation under the principle of detailed balance (or microscopic reversibility) from nonresonant PICSs.

\subsection{Rate coefficients}

The RR rate coefficient is obtained by

$\alpha_{i}(T)=\int_{0}^{\infty} v \sigma_{i}(v) f(v, T) \mathrm{d} v$,

where $v$ is the velocity of the recombining electron, $\sigma_{i}$ is the individual detailed (level/term/shell-resolved) RRCS, $f(v, T)$ is the probability density distribution of the velocity of the recombining electrons for the electron temperature $T$. The Maxwell-Boltzmann distribution for the free electrons is adopted throughout the calculation, with the same quadrature approach as described in Badnell (2006). Accordingly, the total RR rate per ion/atom is

$\alpha_{\mathrm{t}}(T)=\sum_{i} \alpha_{i}(T)$

Total RR rates for all the isoelectronic sequences, taking contributions up to $n=10^{3}$ into account (see its necessity in Sect. 3).

The RR electron energy loss rate coefficient is defined as (e.g., Osterbrock 1989)

$\beta_{i}(T)=\frac{1}{k T} \int_{0}^{\infty} \frac{1}{2} m v^{3} \sigma_{i}(v) f(v, T) \mathrm{d} v$,

The total electron energy loss rate due to RR is obtained simply by adding all the contributions from individual captures,

$L_{\mathrm{t}}(T)=\sum_{i} L_{i}=k T \sum_{i} \beta_{i}$, 
which can be identically derived via

$L_{\mathrm{t}}(T)=k T \alpha_{\mathrm{t}}(T) f_{\mathrm{t}}(T)$,

where

$f_{\mathrm{t}}(T)=\frac{\sum_{i} \beta_{i}(T)}{\alpha_{\mathrm{t}}(T)}$,

is defined as the weighted electron energy loss factor (dimensionless) hereafter.

The above calculation of the electron energy loss rates is realized by adding Eq. (3) into the archival post-processor FORTRAN code ADASRR ${ }^{2}$ (v1.11). Both the level-resolved and bundled- $n / n l$ RR data and the RR electron energy loss data are obtained. The output files have the same format of adf 48 with RR rates and electron energy loss rates in the units of $\mathrm{cm}^{3} \mathrm{~s}^{-1}$ and ryd $\mathrm{cm}^{3} \mathrm{~s}^{-1}$, respectively. Ionization potentials of the ground level of the recombined ions from NIST $^{3}$ (v5.3) are adopted to correct the conversion from PICSs to RRCSs at low kinetic energy for low-charge ions. We should point out that the level-resolved and bundled- $n l / n$ RR data are, in fact, available on OPEN $\mathrm{ADAS}^{4}$, given the fact that we use the latest version of the AUTOSTRUCTURE code and a modified version of the ADASRR code, here we recalculate the RR data, which are used together with the RR electron energy loss data to derive the weighted electron energy loss factor $f_{\mathrm{t}}$ for consistency. In general, our re-calculate RR data are almost identical to those on OPEN ADAS, except for a few many-electron ions at the the high temperature end, where our recalculated data differ by a few percent. Whereas, both RR data and electron energy loss data are a few orders of magnitude smaller compared to those at the lower temperature end, thus, the above-mentioned difference has negligible impact on the accuracy of the weighted electron energy loss factor (see also in Sect. 4.4).

For all the isoelectronic sequences discussed here, the conventional ADAS 19-point temperature grid $z^{2}\left(10-10^{7}\right) \mathrm{K}$ is used.

\section{Results}

For each individual capture due to radiative recombination, when $k T \ll I$, where $I$ is the ionization potential, the RR electron energy loss rate $L_{i}$ is nearly identical to $k T \alpha_{i}$, since the Maxwellian distribution drops exponentially for $E_{\mathrm{k}} \gtrsim k T$, where $E_{\mathrm{k}}$ is the kinetic energy of the free electron before recombination. On the other hand, when $k T \gg I$, the RR electron energy loss rate is negligible compared with $k T \alpha_{i}$. As in an electron-ion collision, when the total energy in the incident channel nearly equals that of a closed-channel discrete state, the channel interaction may cause the incident electron to be captured in this state (Fano \& Cooper 1968). That is to say, those electrons with $E_{\mathrm{k}} \simeq$ $I$ are preferred to be captured, thus, $L_{i} \sim I \alpha_{i}$. Figure 1 shows the ratio of $\beta_{i} / \alpha_{i}=L_{i} /\left(k T \alpha_{i}\right)$ for representative $n L S J$-resolved levels (with $n \leq 8$ ) of He-like Mg XI .

In terms of capturing free electrons into individual shells (bundled- $n$ ), owing to the rapid decline of the ionization potentials for those very high- $n$ shells, the ionization potentials can be comparable to $k T$, if not significantly less than $k T$, at the low temperature end. Therefore we see the significant difference

\footnotetext{
2 http://amdpp.phys.strath.ac.uk/autos/ver/misc/ adasrr. $f$

3 http://physics.nist.gov/PhysRefData/ASD/ionEnergy. html

4 http://open.adas.ac.uk/adf48
}

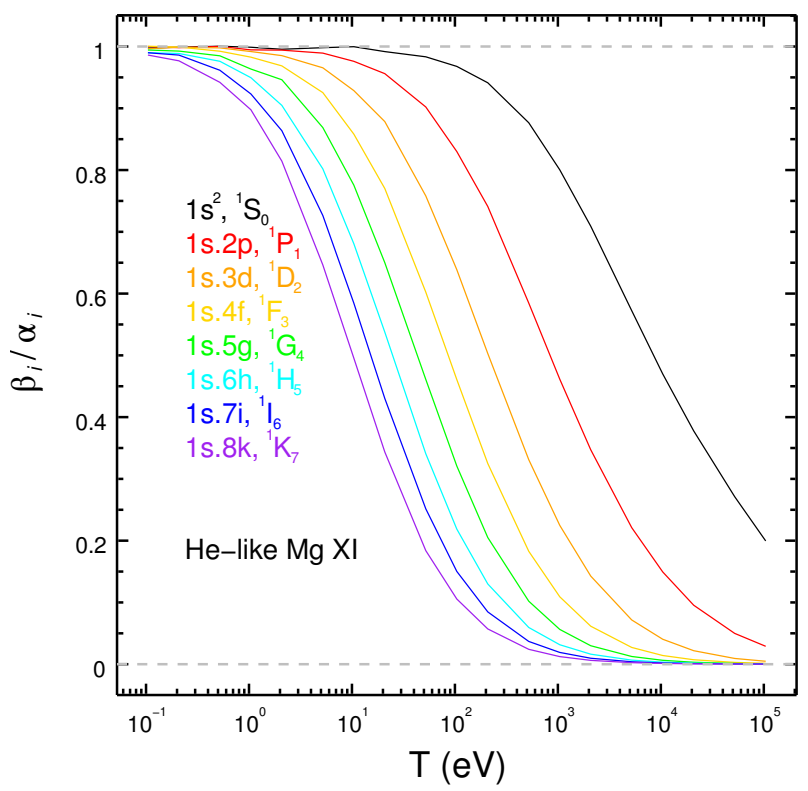

Fig. 1. For He-like Mg XI, the ratio between level-resolved electron energy loss rates $L_{i}$ and the corresponding radiative recombination rates times the temperature of the plasma, i.e. $\beta_{i} / \alpha_{i}$ (not be confused with $\beta_{i} / \alpha_{\mathrm{t}}$ ), where $i$ refers to the $n L S J$-resolved levels with $n \leq 8$ (shown selectively in the plot).

between the top panel (low- $n$ shells) and middle panel (high- $n$ shells) of Fig. 2. In order to achieve adequate accuracy, contributions from high- $n$ shells (up to $n \leq 10^{3}$ ) ought to be included. The middle panel of Fig. 2 shows clearly that even for $n=999$ (the line at the bottom), at the low temperature end, the ratio between $\beta_{n=999}$ and $\alpha_{n=999}$ does not drop to zero. Nevertheless, the bottom panel of Fig. 2 illustrates the advantage of weighting the electron energy loss rate coefficients with respect to the total RR rates, i.e. $\beta_{i} / \alpha_{\mathrm{t}}$, which approaches zero more quickly. At least, for the next few hundred shells following $n=999$, their weighted electron energy loss factors should be no more than $10^{-5}$, thus, their contribution to the total electron energy loss rate should be less than $1 \%$.

The bottom panels of Figs. 3 and 4 illustrate the weighted electron energy loss factors for He-like isoelectronic sequences $(\mathrm{He}, \mathrm{Si}$ and $\mathrm{Fe})$ and $\mathrm{Fe}$ isonuclear sequence $(\mathrm{H}-, \mathrm{He}-, \mathrm{Be}-$ and $\mathrm{N}$-like), respectively. The deviation from (slightly below) unity at the lower temperature end is simply because the weighted electron energy loss factors of the very high- $n$ shells are no longer close to unity (Fig. 2, middle panel). The deviation from (slightly above) zero at the high temperature end occurs because the ionization potentials of the first few low- $n$ shells can still be comparable to $k T$, while sum of these $n$-resolved RR rates are more or less a few tens of percent of the total RR rates.

Because of the nonhydrogenic screening of the wave function for low- $n l$ states in low-charge many-electron ions, the characteristic high-temperature bump is present in not only the RR rates (see Fig. 4 in Badnell 2006, for an example) but also in the electron energy loss rates. The feature is even enhanced in the weighted electron energy loss factor.

We parameterize the ion/atom-resolved radiative recombination electron energy loss factors using the same fitting strategy described in Mao \& Kaastra (2016) with the model function of

$f_{\mathfrak{t}}(T)=a_{0} T^{-b_{0}-c_{0} \log T}\left(\frac{1+a_{2} T^{-b 2}}{1+a_{1} T^{-b 1}}\right)$, 


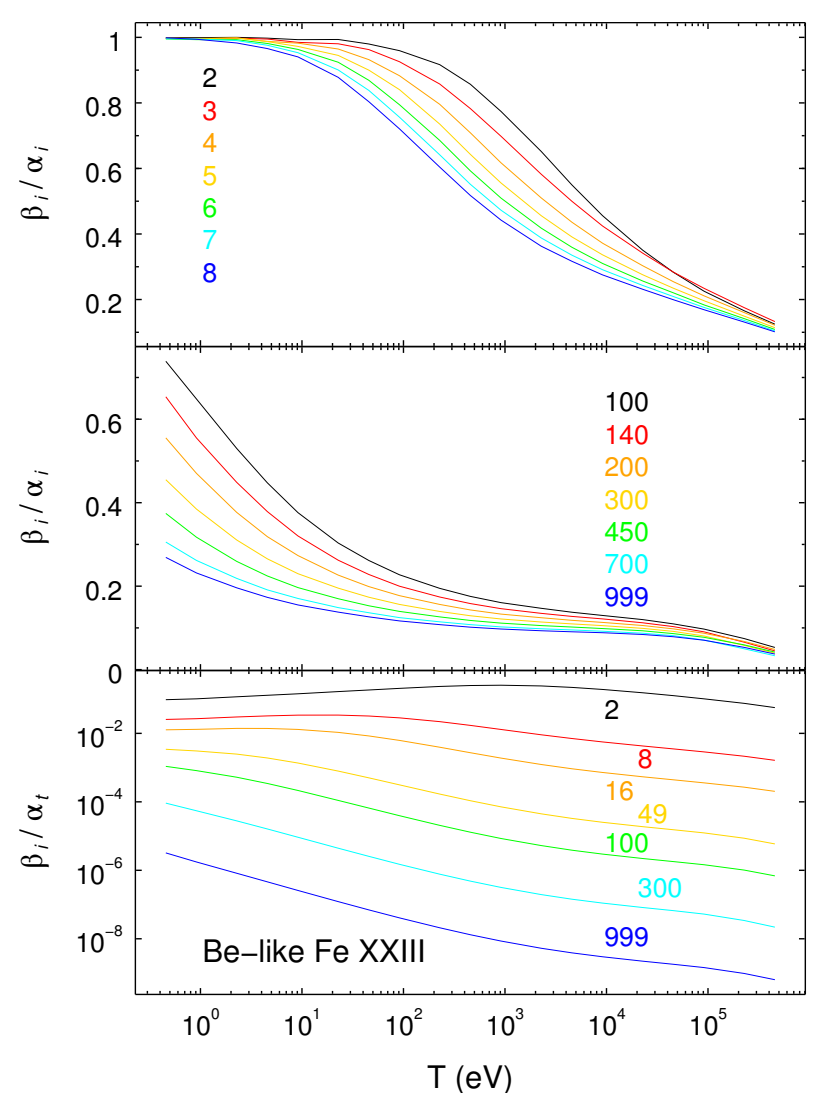

Fig. 2. Ratios of $\beta_{i} / \alpha_{i}$ for Be-like Fe XXIII (upper and middle panel) and ratios of $\beta_{i} / \alpha_{\mathrm{t}}$ (bottom panel), where $i$ refers to the shell number. Lowand high- $n$ shell results are shown selectively in the plot. The upper panel shows all the shells with $n \leq 8$. The middle panel shows shells with $n=100,140,200,300,450,700$, and 999. In the lower panel the shells are $n=2,8,16,49,100,300$, and 999 .

where the electron temperature $T$ is in units of $\mathrm{eV}, a_{0}$ and $b_{0}$ are primary fitting parameters, and $c_{0}, a_{1,2}$, and $b_{1,2}$ are additional fitting parameters. The additional parameters are frozen to zero if they are not used. Furthermore, we constrain $b_{0-2}$ to be within -10.0 to 10.0 and $c_{0}$ between 0.0 and 1.0. The initial values of the two primary fitting parameters $a_{0}$ and $b_{0}$ are set to unity together with the four additional fitting parameters $a_{1,2}$ and $b_{1,2}$ if they are thawed. Conversely, the initial value of $c_{0}$, if it is thawed, is set to either side of its boundary, i.e., $c_{0}=0.0$ or $c_{0}=1.0$ (both fits are performed).

In order to estimate the goodness of fit, the fits are performed with a set of artificial relative errors $(r)$. We started with $r=0.625 \%$, following with increasing the artificial relative error by a factor of two, up to and including $2.5 \%$. The chi-squared statistics adopted here are

$\chi^{2}=\sum_{i=1}^{N}\left(\frac{n_{i}-m_{i}}{r n_{i}}\right)^{2}$,

where $n_{i}$ is the $i$ th numerical calculation result and $m_{i}$ is the $i$ th model prediction (Eq. (7)).

For the model selection, we first fit the data with the simplest model (i.e. all the five additional parameters are frozen to zero), following with fits with free additional parameters step by step. Thawing one additional parameter decreases the degrees of freedom by one. Thus, the more complicated model is only favored (at a 90\% nominal confidence level) if the obtained statistics $\left(\chi^{2}\right)$

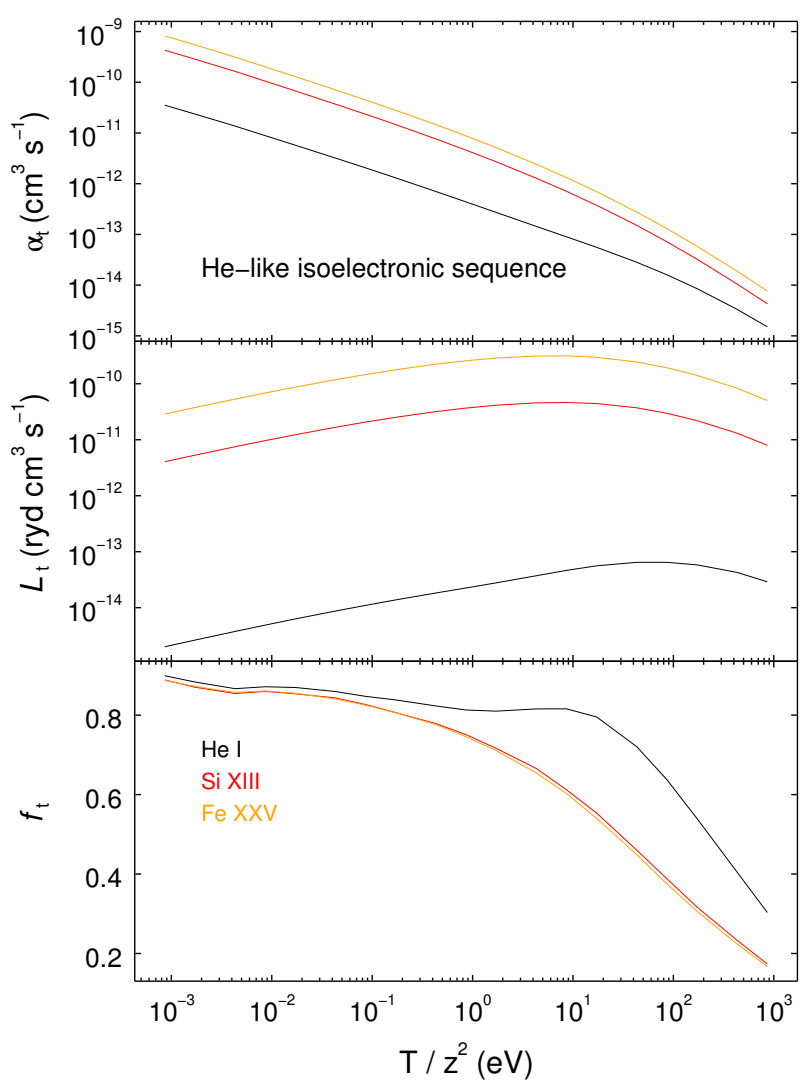

Fig. 3. Total RR rates $\alpha_{\mathrm{t}}$ (top), electron energy loss rates $L_{\mathrm{t}}$ (middle) and weighted electron energy loss factors $f_{\mathrm{t}}$ (bottom) of He-like isoelectronic sequences for ions, including He I (black), Si XIII (red) and Fe XXV (orange). The temperature is downscaled by $z^{2}$, where $z$ is the ionic charge of the recombined ion, to highlight the discrepancy between hydrogenic and nonhydrogenic. The captures to form the He I shows nonhydrogenic feature in the bottom panel.

of this model improves by at least $2.71,4.61,6.26,7.79$, and 9.24 for one to five additional free parameter(s), respectively.

Parameterizations of the ion/atom-resolved RR weighted electron energy loss factors for individual ions/atoms in H-like to Ne-like isoelectronic sequences were performed. A typical fit for nonhydrogenic systems is shown in Fig. 5 for N-like iron (Fe XX). The fitting parameters can be found in Table 2. Again, the weighted energy loss factor per ion/atom is close to unity at low temperature end and drops toward zero rapidly at the high temperature end.

In Fig. 6 we show the histogram of maximum deviation $\delta_{\max }$ (in percent) between the fitted model and original calculation for all the ions considered here. In short, our fitting accuracy is within $4 \%$, and is even accurate $(\lesssim 2.5 \%)$ for the more important $\mathrm{H}$-like, He-like and $\mathrm{Ne}$-like isoelectronic sequences.

In addition, we also specifically fit for Case $\mathrm{A}\left(f_{\mathrm{A}}=\beta_{\mathrm{t}} / \alpha_{\mathrm{t}}\right)$ and Case B (Baker \& Menzel 1938, $f_{\mathrm{B}}=\beta_{n \geq 2} / \alpha_{n \geq 2}$ ) the RR weighted electron energy loss factors of $\mathrm{HI}$ (Fig. 7) and $\mathrm{He}$ I (Fig. 8). Typical unparameterized factors $\left(f_{\mathrm{A}}\right.$ and $\left.f_{\mathrm{B}}\right)$ and fitting parameters can be found in Tables 1 and 2, respectively.

\section{Discussions}

\subsection{Comparison with previous results for $\mathrm{HI}$ and $\mathrm{He} I$}

Figure 9 shows a comparison of RR rates $\left(\alpha_{t}^{\mathrm{RR}}\right)$, electron energy loss rates $\left(L_{t}^{\mathrm{RR}}\right)$, weighted electron energy loss factors $\left(f_{t}^{\mathrm{RR}}\right)$ 


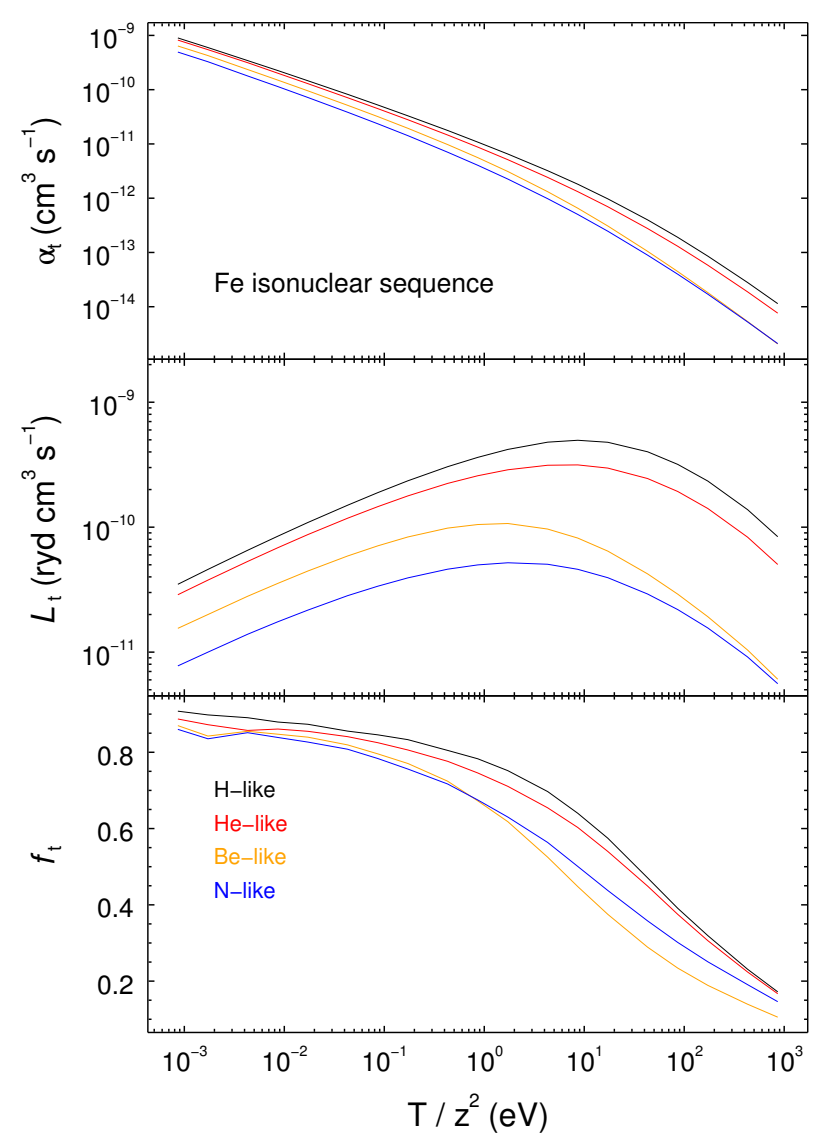

Fig. 4. Top panel is total RR rates $\alpha_{\mathrm{t}}$ of the Fe isonuclear sequence, including $\mathrm{H}$ - (black), $\mathrm{He}$ - (red), $\mathrm{Be}$ - (orange) and $\mathrm{N}$-like (blue); middle panel is the RR electron energy loss rates $L_{t}$; and the bottom panel is the weighted electron energy loss factors $f_{\mathrm{t}}$. The temperature of the plasma is downscaled by $z^{2}$, as in Fig. 3.

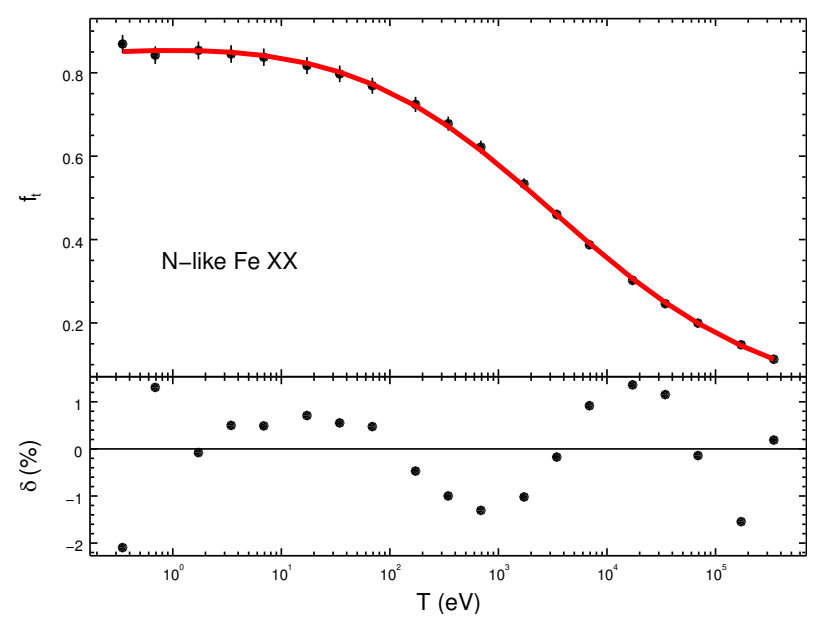

Fig. 5. Radiative recombination weighted electron energy loss factor for $\mathrm{N}$-like iron (Fe XX). The black dots in both panels (associated with artificial error bars of $2.5 \%$ in the upper panel) are the calculated weighted electron energy loss factor. The red solid line is the best fit. The lower panel shows the deviation (in percent) between the best fit and the original calculation.

from this work, Seaton (1959, blue), Ferland et al. (1992, orange) and Hummer (1994, red). Since both Ferland et al. (1992) and Hummer (1994) use the same PICSs (Storey \& Hummer 1991), as expected the two results are highly consistent. The Case A and Case B results of this work are also consistent within

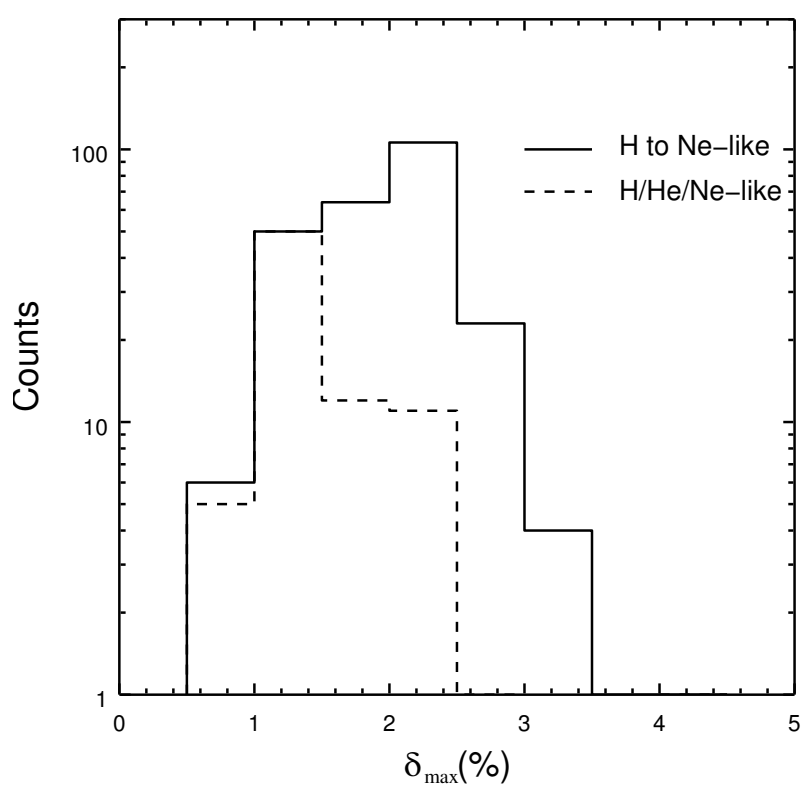

Fig. 6. Histogram of maximum deviation in percent $\left(\delta_{\max }\right)$ for all the ions considered here, which reflects the overall goodness of our parameterization. The dashed histogram is the statistics of the more important $\mathrm{H}$-like, He-like and Ne-like isoelectronic sequences, while the solid histogram is the statistics of all the isoelectronic sequences.

Table 1. Unparameterized of RR weighted electron energy loss factors for $\mathrm{HI}, \mathrm{He} \mathrm{I}$ and Fe XX.

\begin{tabular}{cccccc}
\hline \hline $\begin{array}{c}T / z^{2} \\
\text { K }\end{array}$ & $\begin{array}{c}\text { H I } \\
\text { Case A }\end{array}$ & $\begin{array}{c}\text { H I } \\
\text { Case B }\end{array}$ & $\begin{array}{c}\text { He I } \\
\text { Case A }\end{array}$ & $\begin{array}{c}\text { He I } \\
\text { Case B }\end{array}$ & $\begin{array}{c}\text { Fe XX } \\
\text { Case A }\end{array}$ \\
\hline $10^{1}$ & 0.911 & 0.895 & 0.899 & 0.882 & 0.869 \\
$10^{2}$ & 0.879 & 0.851 & 0.871 & 0.844 & 0.845 \\
$10^{3}$ & 0.841 & 0.786 & 0.847 & 0.797 & 0.797 \\
$10^{4}$ & 0.780 & 0.668 & 0.813 & 0.701 & 0.678 \\
$10^{5}$ & 0.642 & 0.470 & 0.816 & 0.578 & 0.460 \\
$10^{6}$ & 0.392 & 0.268 & 0.637 & 0.486 & 0.246 \\
$10^{7}$ & 0.172 & 0.123 & 0.303 & 0.265 & 0.113 \\
\hline
\end{tabular}

Notes. For HI and He II, both Case A and Case B results are treated separately. Machine readable unparameterized Case A factors for all the ions considered here are available on CDS.

$1 \%$ at the low temperature end and increase to $\sim 5 \%$ (underestimation). For the high temperature end $(T \gtrsim 0.1 \mathrm{keV})$, since the ion fraction of $\mathrm{HI}$ is rather low (almost completely ionized), the present calculation is still acceptable. A similar issue at the high temperature end is also found in Case A results of Seaton (1959) with a relatively significant overestimation $(\gtrsim 5 \%)$ from the other three calculations.

Likewise, the comparison for He I between this work and Hummer \& Storey (1998) is presented in Fig. 10. The Case A and Case B results from both calculations agree well (within 2\%) at the low temperature end $(T \lesssim 2.0 \mathrm{eV})$. At higher temperatures with $T \gtrsim 2 \mathrm{eV}$, the RR rate and electron energy loss rate for He I are not available in Hummer \& Storey (1998).

\subsection{Scaling with $z^{2}$}

In previous studies of hydrogenic systems, Seaton (1959), Ferland et al. (1992), and Hummer \& Storey (1998), all use $z^{2}$ scaling for $\alpha_{\mathrm{t}}^{\mathrm{RR}}$. That is to say, $\alpha_{\mathrm{t}}^{X}=z^{2} \alpha_{\mathrm{t}}^{\mathrm{H}}$, where $z$ is the ionic 
Table 2. Fitting parameters of RR weighted electron energy loss factors for H I, He I and Fe XX.

\begin{tabular}{|c|c|c|c|c|c|c|c|c|c|c|}
\hline$s$ & $Z$ & Case & $a_{0}$ & $b_{0}$ & $c_{0}$ & $a_{1}$ & $b_{1}$ & $a_{2}$ & $b_{2}$ & $\delta_{\max }$ \\
\hline 1 & 1 & A & $8.655 \mathrm{E}+00$ & $5.432 \mathrm{E}-01$ & $0.000 \mathrm{E}+00$ & $1.018 \mathrm{E}+01$ & $5.342 \mathrm{E}-01$ & $0.000 \mathrm{E}+00$ & $0.000 \mathrm{E}+00$ & $1.2 \%$ \\
\hline 1 & 1 & B & $2.560 \mathrm{E}+00$ & $4.230 \mathrm{E}-01$ & $0.000 \mathrm{E}+00$ & $2.914 \mathrm{E}+00$ & 4.191E-01 & $0.000 \mathrm{E}+00$ & $0.000 \mathrm{E}+00$ & $2.1 \%$ \\
\hline 2 & 2 & A & $2.354 \mathrm{E}+00$ & 3.367E-01 & $0.000 \mathrm{E}+00$ & $6.280 \mathrm{E}+01$ & 8.875E-01 & $2.133 \mathrm{E}+01$ & $5.675 \mathrm{E}-01$ & $1.5 \%$ \\
\hline 2 & 2 & $\mathrm{~B}$ & $1.011 \mathrm{E}+04$ & $1.348 \mathrm{E}+00$ & $4.330 \mathrm{E}-03$ & $1.462 \mathrm{E}+04$ & $1.285 \mathrm{E}+00$ & $0.000 \mathrm{E}+00$ & $0.000 \mathrm{E}+00$ & $3.5 \%$ \\
\hline 7 & 26 & $\mathrm{~A}$ & $2.466 \mathrm{E}+01$ & $4.135 \mathrm{E}-01$ & $0.000 \mathrm{E}+00$ & $2.788 \mathrm{E}+01$ & $4.286 \mathrm{E}-01$ & $0.000 \mathrm{E}+00$ & $0.000 \mathrm{E}+00$ & $2.1 \%$ \\
\hline
\end{tabular}

Notes. For HI and He II, both Case A and Case B results are included. $s$ is the isoelectronic sequence number of the recombined ion, $Z$ is the atomic number of the ion, $a_{0-2}, b_{0-2}$ and $c_{0}$ are the fitting parameters and $\delta_{\max }$ is the maximum deviation (in percent) between the "best-fit" and original calculation. Case A and Case B refers to $\beta_{\mathrm{t}} / \alpha_{\mathrm{t}}$ and $\beta_{n \geq 2} / \alpha_{n \geq 2} \mathrm{RR}$ weighted electron energy loss factors, respectively. Machine readable fitting parameters and maximum deviation (in percent) for the total weighted electron energy loss factors for all the ions considered here are available on CDS.

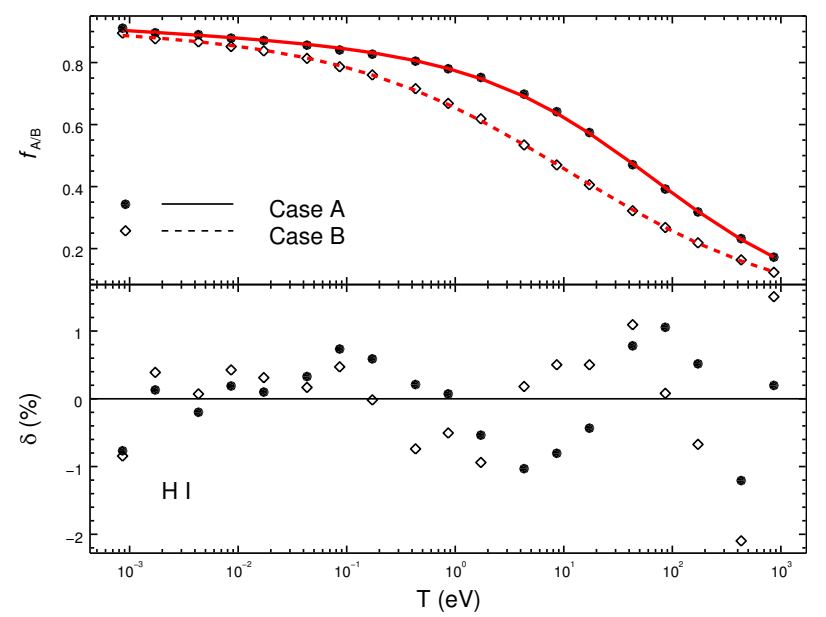

Fig. 7. Case A (solid line, filled circles) and Case B (dashed line, empty diamonds) RR weighted electron energy loss factor $\left(f_{\mathrm{A} / \mathrm{B}}\right)$ for H I. The black dots in both panels (associated with artificial error bars in the upper one) are the calculated weighted electron energy loss factor. The red solid line is the best fit. The lower panel shows the deviation (in percent) between the best fit and the original calculation.

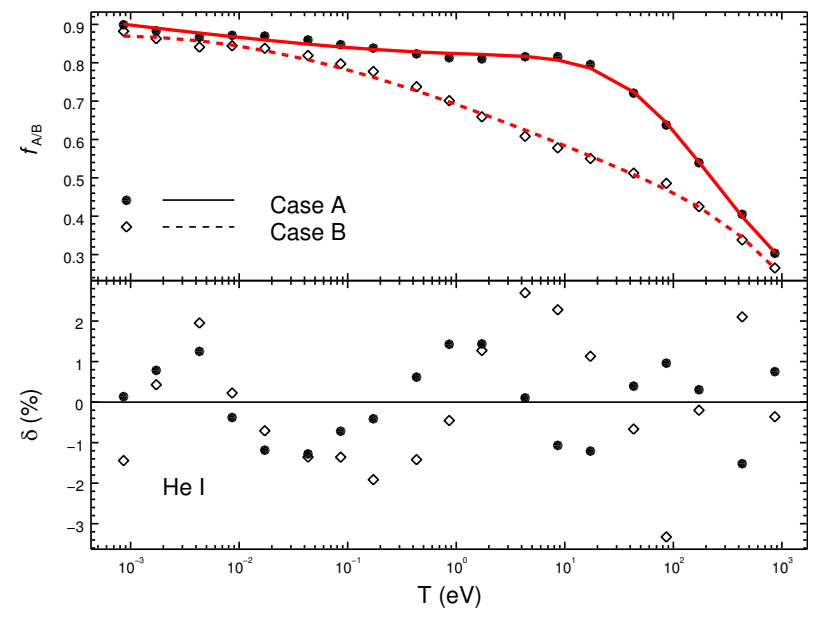

Fig. 8. Similar to Fig. 7 but for He I.

charge of the recombined ion $X$. The same $z^{2}$ scaling also applies for $\beta_{\mathrm{t}}^{\mathrm{RR}}$ (or $L_{\mathrm{t}}^{\mathrm{RR}}$ ). LaMothe \& Ferland (2001) also pointed out that the shell-resolved ratio of $f_{n}^{R R}\left(=\beta_{n}^{\mathrm{RR}} / \alpha_{n}^{\mathrm{RR}}\right)$ can also be scaled with $z^{2} / n^{2}$, i.e., $f_{n}^{X}=\frac{z^{2}}{n^{2}} f_{n}^{\mathrm{H}}$ where $n$ refers to the principle quantum number.

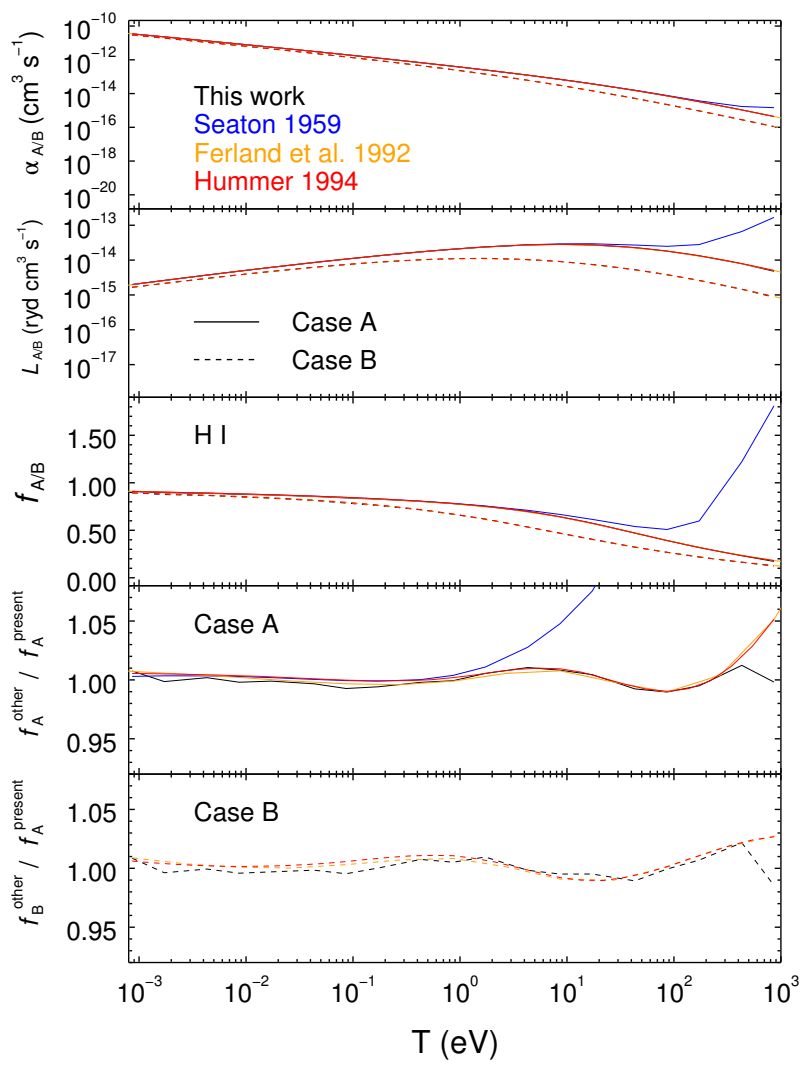

Fig. 9. Comparison of the RR data for $\mathrm{H}$ I among results from this work (black), Seaton (1959, blue), Ferland et al. (1992, orange), and Hummer (1994, red). Both results of case A (solid lines) and case B (dashed lines) are shown. The total RR rates $\left(\alpha_{\mathrm{A} / \mathrm{B}}^{\mathrm{RR}}\right)$ and electron energy loss rates $\left(L_{\mathrm{A} / \mathrm{B}}^{\mathrm{RR}}\right)$ are shown in the top two panels. The RR weighted electron energy loss factors $\left(f_{\mathrm{A} / \mathrm{B}}\right)$ are shown in the middle panel. The ratios of $f_{\mathrm{A} / \mathrm{B}}$ from this work and previous works with respect to the fitting results (Eq. (7) and Table 2) of this work, i.e., $f_{\mathrm{A} / \mathrm{B}}^{\text {other }} / f_{\mathrm{A} / \mathrm{B}}^{\text {prest }}$, are shown in the bottom two panels.

In the following, we merely focus on the scaling for the ion/atom-resolved data set. In the top panel of Fig. 11 we show the ratios of $f_{t} / z^{2}$ for $\mathrm{H}$-like ions. Apparently, from the bottom panel of Fig. 11, the $z^{2}$ scaling for the H-like isoelectronic sequence is accurate within $2 \%$. For the rest of the isoelectronic sequences, for instance, the He-like isoelectronic sequence shown in Fig. 12, the $z^{2}$ scaling applies at the low temperature end, whereas, the accuracies are poorer toward the high temperature end. We also show the $z^{2}$ scaling for the $\mathrm{Fe}$ isonuclear sequence in Fig. 13. 
J. Mao et al.: RR electron energy loss rate

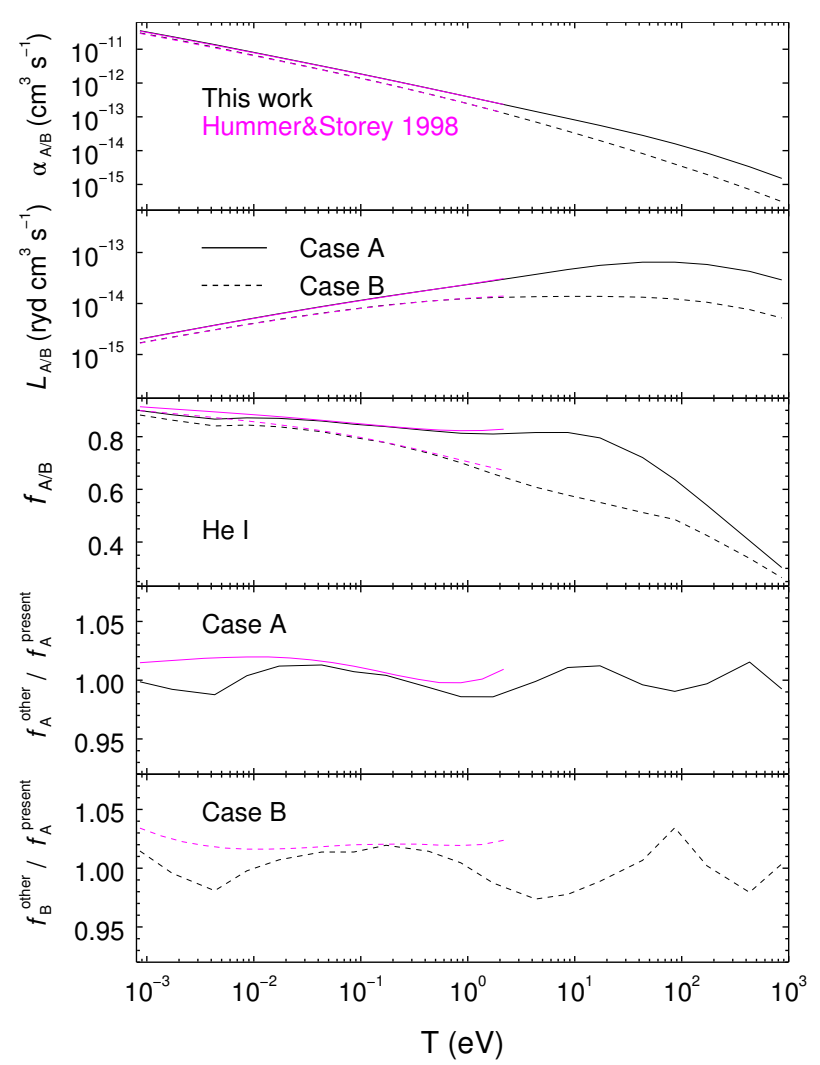

Fig. 10. Similar to Fig. 10 but for He I between this work (black) and Hummer \& Storey (magenta 1998). The latter only provides data with $T \leq 10^{4.4} \mathrm{~K}$.

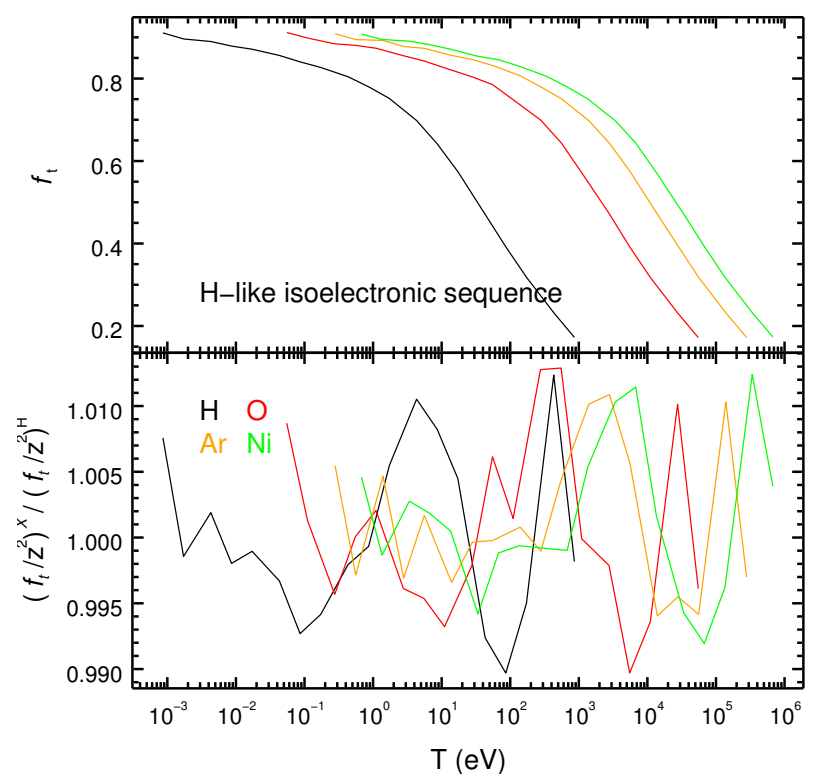

Fig. 11. $z^{2}$ scaling for the H-like isoelectronic sequence (Case A), including HI (black), O VIII (red), Ar XVIII (orange) and Ni XXVIII (green). The top panel shows the ratios of $f_{t} / z^{2}$ as a function of electron temperature $(T)$. The bottom panel is the ratio of $\left(f_{\mathrm{t}} / z^{2}\right)^{X}$ for ion $X$ with respect to the ratio of $\left(f_{\mathrm{t}} / z^{2}\right)^{\mathrm{H}}$ for $\mathrm{H}$.

\subsection{Radiative recombination continua}

We restrict the discussion above to the RR energy loss of the electrons in the plasma. The ion energy loss of the ions due to RR can be estimated as $P^{\mathrm{RR}} \sim I_{i} \alpha_{i}$, where $I_{i}$ is the ionization potential of the level/term into which the free electron is captured,

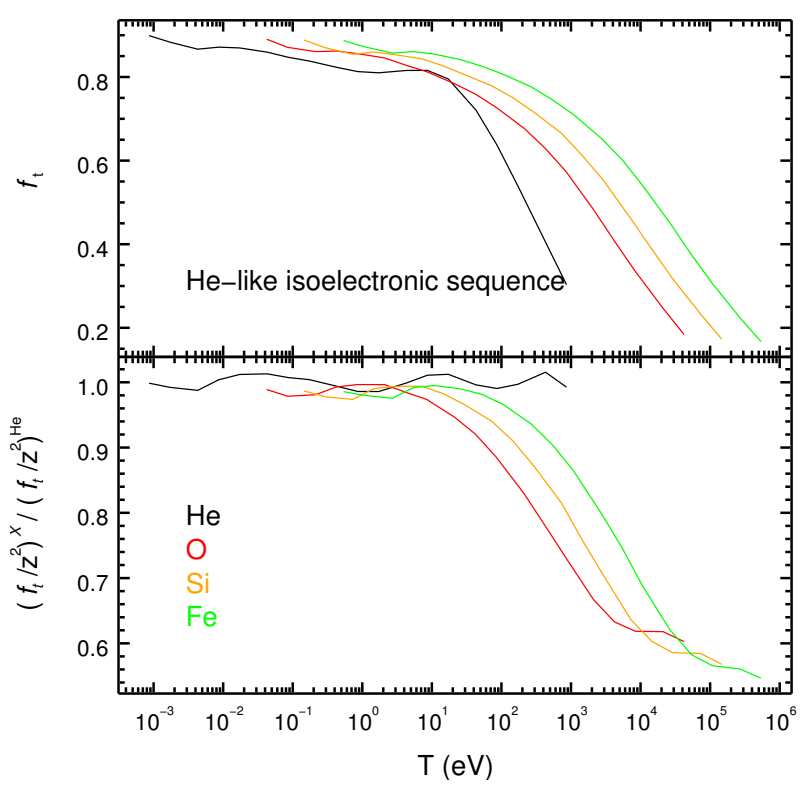

Fig. 12. Similar to Fig. 11 but for the $z^{2}$ scaling for the He-like isoelectronic sequences.

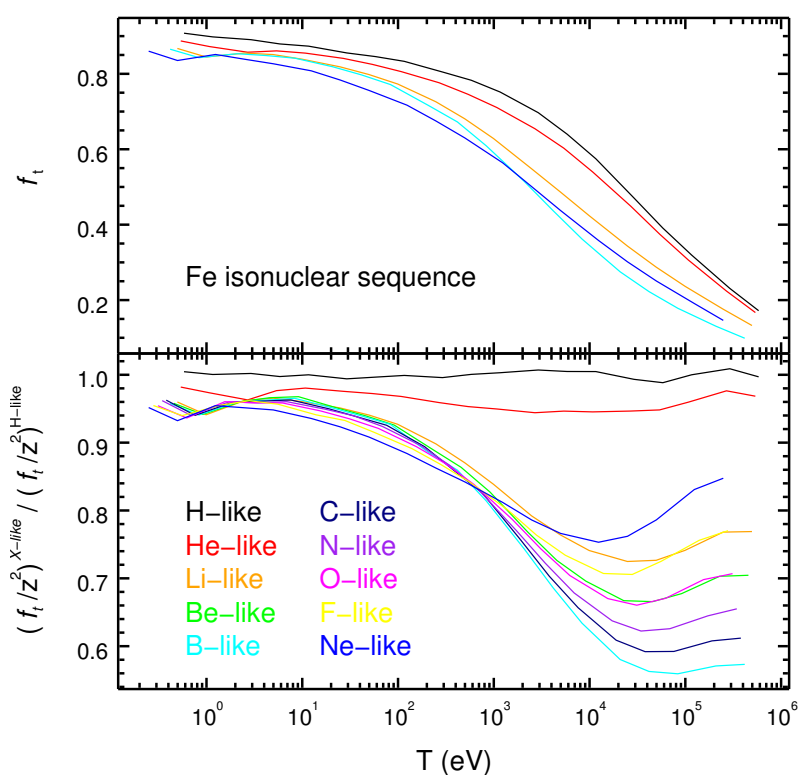

Fig. 13. $z^{2}$ scaling for the Fe isonuclear sequence. The top panel shows the ratios of $f_{\mathrm{t}} / z^{2}$ as a function of electron temperature $(T)$. The bottom panel is the ratio of $\left(f_{\mathrm{t}} / z^{2}\right)^{X \text {-like }}$ for $X$-like Fe with respect to the ratio of $\left(f_{\mathrm{t}} / z^{2}\right)^{\mathrm{H} \text {-like }}$ for H-like Fe XXVI.

and $\alpha_{i}$ is the corresponding RR rate coefficient. Whether to include the ionization potential energies as part of the total internal energy of the plasma is not critical as long as the entire computation of the net energy gain/loss is self-consistent (see a discussion in Gnat \& Ferland 2012). On the other hand, when interpreting the emergent spectrum due to RR, such as the radiative recombination continua ( $R R C$ ) for a low-density plasma, the ion energy loss of the ion is essentially required. The RRC emissivity (Tucker \& Gould 1966) can be obtained via

$$
\begin{aligned}
\frac{\mathrm{d} E^{\mathrm{RRC}}}{\mathrm{d} t \mathrm{~d} V} & =\int_{0}^{\infty} n_{\mathrm{e}} n_{\mathrm{i}}\left(I+\frac{1}{2} m v^{2}\right) v \sigma(v) f(v, k T) \mathrm{d} v \\
& =n_{\mathrm{e}} n_{\mathrm{i}} I\left(1+f_{\mathrm{t}} k T / I\right) \alpha_{\mathrm{t}},
\end{aligned}
$$




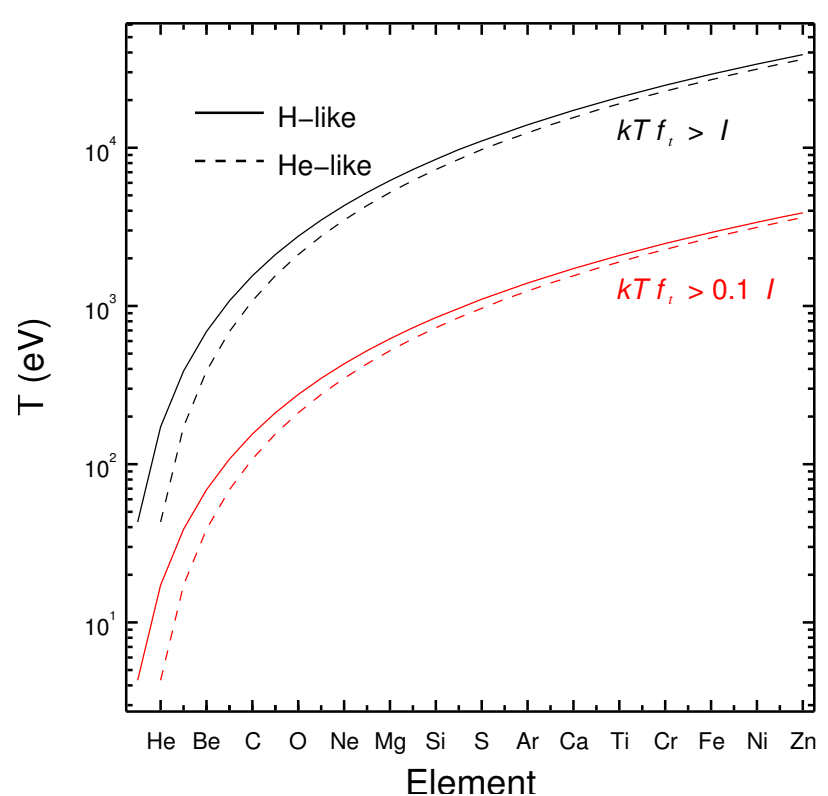

Fig. 14. Threshold temperature above which the electron energy loss via RR cannot be neglected, compared to the ion energy loss, for H-like (solid lines) and He-like ions (dashed lines).

where $n_{\mathrm{e}}$ and $n_{\mathrm{i}}$ are the electron and (recombining) ion number density, respectively. Generally speaking, the ion energy loss of the ion dominates the electron energy loss of the electrons, since $f_{\mathrm{t}}$ is on the order of unity while $k T \lesssim I$ holds for those X-ray photoionizing plasmas in XRBs (Liedahl \& Paerels 1996), AGN (Kinkhabwala et al. 2002) and recombining plasmas in SNRs (Ozawa et al. 2009). Figure 14 shows the threshold temperature above which the electron energy loss via RR cannot be neglected compared to the ion energy loss. For hot plasmas with $k T \gtrsim 2 \mathrm{keV}$, the electron energy loss is comparable to the ion energy loss for $Z>5$. We emphasize that we refer to the electron temperature $T$ of the plasma here, which is not necessarily identical to the ion temperature of the plasma, in particular, in the nonequilibrium ionization scenario.

\subsection{Total radiative recombination rate}

Various calculations of (total or shell/term/level-resolved) RR data are available from the literature. Historically, different approaches have been used for calculating the total RR rates, including the Dirac-Hartree-Slater method (Verner et al. 1993) and the distorted-wave approximation ( Gu 2003; Badnell 2006). Additionally, Nahar and coworkers (e.g., Nahar 1999) obtained the total (unified DR $+\mathrm{RR}$ ) recombination rate for various ions with their $\boldsymbol{R}$-matrix calculations. Different approaches can lead to different total RR rates (see a discussion in Badnell 2006) as well as the individual term/level-resolved RR rate coefficients, even among the most advanced $\boldsymbol{R}$-matrix calculations. Nevertheless, the bulk of the total RR rates for various ions agrees well among each other. As for the detailed RR rate coefficients, and consequently, the detailed RR electron energy loss rate, the final difference in the total weighted electron energy loss factors $f_{\mathrm{t}}$ are still within $1 \%$, as long as the difference among different methods are within a few percent and given the fact that each individual RR is $\lesssim 10 \%$ of the total RR rate for a certain ion/atom. In other words, although we used the recalculated total RR rate (Sect. 2.2) to derive the weighted electron energy loss factors, we assume these factors can still be applied to other total RR rates.

Acknowledgements. J.M. acknowledges discussions and support from M. Mehdipour, A. Raassen, L. Gu, and M. O'Mullane. We thank the referee, G. Ferland, for valuable comments on the manuscript. SRON is supported financially by NWO, the Netherlands Organization for Scientific Research.

\section{References}

Badnell, N. R. 1986, J. Phys. B At. Mol. Phys., 19, 3827

Badnell, N. R. 2006, ApJS, 167, 334

Badnell, N. R., \& Seaton, M. J. 2003, J. Phys. B At. Mol. Phys., 36, 4367

Baker, J. G., \& Menzel, D. H. 1938, ApJ, 88, 52

Burgess, A. 1965, MmRAS, 69, 1

Cox, D. P., \& Daltabuit, E. 1971, ApJ, 167, 113

Hummer, D. G. 1994, MNRAS, 268, 109

Hummer, D. G., \& Storey, P. J. 1998, MNRAS, 297, 1073

Fano, U., \& Cooper, J. W. 1968, Rev. Mod. Phys., 40, 441

Ferland, G. J., Peterson, B. M., Horne, K., Welsh, W. F., \& Nahar, S. N. 1992, ApJ, 387, 95

Ferland, G. J., Korista, K. T., Verner, D. A., et al. 1998, PASP, 110, 761

Ferland, G. J., Porter, R. L., van Hoof, P. A. M., et al. 2013, Rev. Mex. Astron. Astrofis., 49, 137

Foster, A. R., Ji, L., Smith, R. K., \& Brickhouse, N. S. 2012, ApJ, 756, 128

Gnat, O., \& Ferland, G. J. 2012, ApJS, 199, 20

Gu, M. F. 2003, ApJ, 589, 1085

Kaastra, J. S., Mewe, R., \& Nieuwenhuijzen, H. 1996, 11th Colloquium on UV and X-ray Spectroscopy of Astrophysical and Laboratory Plasmas, 411

Kaastra, J. S., Bykov, A. M., Schindler, S., et al. 2008, Space Sci. Rev., 134, 1

Kallman, T. R., \& McCray, R. 1982, ApJS, 50, 263

Kim, Y. S., \& Pratt, R. H. 1983, Phys. Rev. A, 27, 2913

Kinkhabwala, A., Sako, M., Behar, E., et al. 2002, ApJ, 575, 732

LaMothe, J., \& Ferland, G. J. 2001, PASP, 113, 165

Liedahl, D. A., \& Paerels, F. 1996, ApJ, 468, L33

Lykins, M. L., Ferland, G. J., Porter, R. L., et al. 2013, MNRAS, 429, 3133

Mao, J., \& Kaastra, J. 2016, A\&A, 587, A84

Nahar, S. N. 1999, ApJS, 120, 131

Osterbrock, D. E. 1989, Research supported by the University of California, John Simon Guggenheim Memorial Foundation, University of Minnesota, et al. Mill Valley, CA, University Science Books, 422

Ozawa, M., Koyama, K., Yamaguchi, H., Masai, K., \& Tamagawa, T. 2009, ApJ, 706, L71

Raymond, J. C., Cox, D. P., \& Smith, B. W. 1976, ApJ, 204, 290

Schure, K. M., Kosenko, D., Kaastra, J. S., Keppens, R., \& Vink, J. 2009, A\&A, 508,751

Seaton, M. J. 1959, MNRAS, 119, 81

Seaton, M. J., Zeippen, C. J., Tully, J. A., et al. 1992, Rev. Mex. Astron. Astrofis., 23, 19

Smith, R. K., Brickhouse, N. S., Liedahl, D. A., \& Raymond, J. C. 2001, ApJ, 556, L91

Storey, P. J., \& Hummer, D. G. 1991, Comput. Phys. Comm., 66, 129

Sutherland, R. S., \& Dopita, M. A. 1993, ApJS, 88, 253

Tucker, W. H., \& Gould, R. J. 1966, ApJ, 144, 244

Verner, D. A., Yakovlev, D. G., Band, I. M., \& Trzhaskovskaya, M. B. 1993, Atomic Data and Nuclear Data Tables, 55, 233

Verner, D. A., \& Ferland, G. J. 1996, ApJS, 103, 467 